\title{
DISBIOSE INTESTINAL E SÍNDROME DO INTESTINO IRRITÁVEL: EFEITO DE UMA DIETA BAIXA EM FODMAPS
}

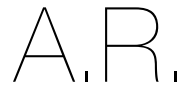
ARTIGO DE REVISÃO

1 Escola Superior de Tecnologia da Saúde do Instituto Politécnico de Coimbra,

Rua 5 de Outubro, São Martinho do Bispo, 3046-854 Coimbra Portugal

"Endereço para correspondência:

Ana Teresa Valadar Martins Rua dos Pescadores, lote 15, 2. Mirandela, 5370-421 anavaladar@gmail.com

Histórico do artigo:

Recebido a 5 de maio de 2020 Aceite a 23 de setembro de 2020

\author{
INTESTINAL DYSBIOSIS AND IRRITABLE BOWEL SYNDROME: \\ EFFECT OF A LOW DIET ON FODMAPS
}

Ana Martins $^{1 *}$; Ana Pequito; Ana Lúcia Baltazar'

\section{RESUMO}

A síndrome do intestino irritável é um distúrbio gastrointestinal multifatorial, que afeta entre 10\% a 25\% da população mundial. 0 diagnóstico da síndrome do intestino irritável, segundo os Critérios de Roma IV, pode ser feito quando o individuo sofre de dor abdominal recorrente e esta está associada a pelo menos dois dos seguintes sintomas: dor relacionada à defecação, alteração na frequência das fezes e alteração na aparência das fezes. Evidências sugerem que uma distorção na biodiversidade e composição da microbiota intestinal, disbiose, interfere na integridade do intestino desempenhando um fator importante no desencadear da síndrome do intestino irritável.

FODMAP é o acrónimo para "oligo-, di- e monossacarídeos e polióis fermentáveis" e designa um grupo de hidratos de carbono de cadeia curta, osmoticamente ativos e altamente fermentáveis no cólon uma vez que a sua absorção é incompleta no intestino delgado. Uma baixa absorção de FODMAPs no intestino delgado pode dever-se a saturação, inibição e defeitos ao nível dos transportadores pelo epitélio (frutose), diminuição da atividade das hidrólases (lactose), deficits enzimáticos (frutanos, galactanos) ou a moléculas de elevadas dimensões incapazes de ser absorvidas por difusão simples (polióis).

A dieta baixa em FODMAPs é constituída por três fases: restrição, reintrodução e manutenção e segundo alguns investigadores, pode ser recomendada como terapia de primeira linha para indivíduos com síndrome do intestino irritável. Estudos comprovam que uma ingestão de até $0,5 \mathrm{~g}$ de FODMAPs (com exceção da lactose) por refeição se mostra eficaz no alívio de sintomas, em $70 \%$ dos doentes. A adoção de uma dieta baixa em FODMAPs reduz os prebióticos e os substratos fermentáveis disponíveis podendo alterar negativamente a estrutura e função da microbiota intestinal.

A prescrição deste regime alimentar não é fácil sendo necessária a intervenção de um nutricionista com conhecimento nesta área, possibilitando o empowerment do doente, o controlo a longo prazo dos sintomas e melhoria da qualidade de vida.

\author{
PALAVRAS-CHAVE \\ Dieta baixa em FODMAPs, Disbiose, FODMAP, Microbiota intestinal, Síndrome do intestino irritável
}

ABSTRACT

Irritable bowel syndrome (IBS) is a multifactorial gastrointestinal disorder, affecting $10 \%$ to $25 \%$ of the world population. The diagnosis of IBS, according to the Rome IV Criteria, can be made when the individual suffers from recurrent abdominal pain and this is associated with at least two of the following symptoms: pain related to defecation, changes in stool frequency, and changes in the appearance of feces. Evidence suggests that a distortion in the biodiversity and composition of the intestinal microbiota, dysbiosis, interferes with the integrity of the intestine playing an important factor triggering IBS.

FODMAP is the acronym for "oligo-, di- and monosaccharides and fermentable polyols". It designates a group of short-chain carbohydrates, osmotically active and highly fermentable in the colon since their absorption is incomplete in the small intestine. Low absorption of FODMAPs in the small intestine may be due to saturation, inhibition, and defects at the level of transporters by the epithelium (fructose), decreased activity of hydrolases (lactose), enzymatic deficits (fructans, galactans), or too large molecules unable to be absorbed by simple diffusion (polyols)

The low FODMAPs diet consists of three phases: restriction, reintroduction, and maintenance, and according to some researchers, it can be recommended as first-line therapy for individuals with IBS. Studies show that an intake of up to 0.5 g of FODMAPs (except for lactose) per meal is effective in relieving symptoms in $70 \%$ of patients.

The adoption of a diet low in FODMAPs reduces prebiotics and available fermentable substrates, which can negatively alter the structure and function of the intestinal microbiota.

The prescription of this diet is not easy, requiring the intervention of a nutritionist with knowledge in this area, enabling patient empowerment, long-term control of symptoms, and improvement in the quality of life.

KEYWORDS

Low FODMAPs diet, Dysbiosis, FODMAP, Intestinal microbiota, Irritable bowel syndrome 


\section{INTRODUÇÃO}

A síndrome do intestino irritável (SII) é um distúrbio gastrointestinal multifatorial. Embora a sua etiologia ainda não seja totalmente compreendida, várias alterações fisiopatológicas estão associadas, tais como motilidade intestinal alterada, hipersensibilidade visceral, inflamação intestinal de baixo grau, aumento da permeabilidade intestinal, desregulação do eixo cérebro-intestino e microbiota intestinal alterada sendo também a predisposição genética um fator associado a este distúrbio (1-6).

Este é um dos distúrbios gastrointestinais mais comuns em todo o mundo, afetando entre 10\% a 25\% da população mundial sendo mais frequente em mulheres e apresentando uma prevalência menor para indivíduos acima de 50 anos (2-4, 7-9).

O diagnóstico da SIl é feito primariamente a partir dos Critérios de Roma IV. Segundo estes, o diagnóstico pode ser feito quando o individuo sofre de dor abdominal recorrente, em média, pelo menos 1 dia por semana durante os últimos 3 meses, com início há pelo menos 6 meses, estando esta associada a pelo menos dois dos seguintes sintomas: dor relacionada à defecação, alteração na frequência das fezes e alteração na aparência das fezes. Todos os casos diagnosticados são posteriormente atribuídos a um subtipo de SII dependendo dos hábitos intestinais, como obstipação (SII-O), diarreia (SII-D), combinação de obstipação e diarreia (SII-M) e indefinida (SII-I) podendo, cada um deles associar-se também aos seguintes sintomas: distensão abdominal e/ou flatulência excessiva. Alguns dos sintomas digestivos mais frequentes associados à SII incluem saciedade precoce, dispepsia intermitente e náusea (7-10).

\section{Disbiose Intestinal e Síndrome do Intestino Irritável}

Evidências sugerem que uma distorção na biodiversidade e composição da microbiota intestinal, denominada disbiose, interfere na integridade do intestino desempenhando um fator importante no desencadear da SII. Esta distorção pode dever-se a fatores como a idade, o tempo de trânsito intestinal e o seu pH, a disponibilidade de substrato fermentável, estado imunológico e o uso de antibióticos $(9,11,12)$

A microbiota gastrointestinal humana representa um ecossistema metabolicamente ativo e complexo onde coabitam milhares de microrganismos, desempenhando funções (imunológicas, metabólicas e motoras) essenciais para a saúde e sobrevivência do hospedeiro. Desta forma, a microbiota atua como uma barreira contra microrganismos invasores promovendo a imunidade intestinal, prevenindo também a proliferação de bactérias patogénicas. Contribui para o metabolismo de nutrientes e vitaminas essenciais, auxiliando na fermentação de hidratos de carbono mal absorvidos ou resistentes ao processo digestivo, favorecendo uma digestão e absorção eficaz de nutrientes. Assim, o comprometimento da sua integridade pode modificar significativamente a fisiologia gastrointestinal e acionar os mecanismos subjacentes à SII $(1,9,11)$.

Estudos científicos comprovam que a microbiota de doentes com SII apresenta uma diminuição global e menor diversidade em comparação com a de indivíduos saudáveis. Evidenciam ainda que bactérias potencialmente perigosas se encontram em concentrações mais elevadas em indivíduos com SII. O crescimento exagerado destas bactérias vai perturbar a função gastrointestinal, desequilibrando a produção das secreções e consequente produção de enzimas pelos órgãos que compõem o trato gastrointestinal $(9,11)$, podendo levar a um défice nutricional uma vez que a capacidade de absorção de nutrientes se encontra diminuída.

\section{O conceito FODMAPs}

FODMAP é o acrónimo para "oligo-, di- e monossacarídeos e polióis fermentáveis" e designa um grupo de hidratos de carbono de cadeia curta (frutanos, galactanos, lactose, frutose e polióis), osmoticamente ativos e altamente fermentáveis no cólon uma vez que a sua absorção é incompleta no intestino delgado $(5,7,13)$.

\section{Frutanos e Galactanos}

Os oligossacarídeos, frutanos e galactanos, não sendo digeridos no trato digestivo devido a défices enzimáticos, atravessam o trato gastrointestinal até atingir o intestino grosso onde são fermentados, com consequente formação de gases e ácidos gordos de cadeia curta $(4,14)$. Estes hidratos de carbono apresentam função de prebióticos uma vez que estimulam o crescimento e a atividade bacteriana de Bifidobacteria e Lactobacilli, potencialmente benéficas (4). Os frutanos são polímeros de frutose ligados a uma molécula de glicose no final e os galactanos são polímeros de galactose. Estes oligossacarídeos apresentam o maior comprimento de cadeia quando comparados com os restantes FODMAPs $(10,14)$.

\section{Lactose}

A lactose é um dissacarídeo constituído pela junção de glicose e galactose e dependente da enzima lactase, presente nas vilosidades do intestino delgado, para a sua hidrolisação e posterior absorção (4, 14, 15). A quantidade disponível desta enzima no intestino está sujeita a fatores genéticos e étnicos ou até de condições gastrointestinais (14). Quando esta enzima se encontra inativa ou numa concentração insuficiente, a lactose não absorvida no intestino delgado é mais tarde utilizada pelas bactérias como substrato fermentável no cólon distal, produzindo ácidos gordos de cadeia curta, hidrogénio e metano em excesso $(10,15)$. Uma ingestão superior a $7 \mathrm{~g}$ de lactose está associada a sintomas decorrentes da sua má absorção (14).

\section{Frutose}

Este monossacarídeo de 6 carbonos pode ser absorvido através de várias vias de transporte facilitado no epitélio intestinal. Quando a frutose está presente na sua forma livre, ou seja, enquanto monossacarídeo, é absorvida por difusão facilitada através do transportador de glicose 5 (GLUT-5), localizado na membrana apical dos enterócitos $(4,14)$. Como a frutose livre é absorvida na sua maioria por mecanismos mediados por transportadores de baixa capacidade, quanto maior for o excesso deste monossacarídeo, maior a probabilidade de ocorrer má absorção. A absorção deste açúcar também pode ser mediada pelo transportador de glicose 2 (GLUT-2) quando se encontra na presença de glicose e galactose podendo ser minimizada na presença de sorbitol. Assim, o excesso de consumo de frutose, a inibição de GLUT-2 ou outros problemas podem resultar na acumulação de frutose não absorvida no intestino grosso. Quantidades de frutose superiores a 25 g não são bem absorvidas por metade da população, sendo que desta má absorção resulta um aumento da carga osmótica no lúmen intestinal e consequente fermentação $(14,16)$.

\section{Polióis}

Sorbitol, manitol, lactitol, xilitol, maltitol e isomalte são polióis (álcoois de açúcar) parcialmente digeridos e absorvidos no intestino delgado e fermentados no intestino grosso $(4,14)$. Quando ingeridos em excesso, por exemplo numa quantidade superior a $50 \mathrm{~g}$ de sorbitol por dia ou superior a $20 \mathrm{~g}$ de manitol por dia, podem desencadear sintomas gastrointestinais (14). 
Mecanismos e Consequências da Má Absorção dos FODMAPs Uma baixa absorção de FODMAPs no intestino delgado pode dever-se a diversos mecanismos como saturação, inibição e defeitos ao nível dos transportadores pelo epitélio (frutose), diminuição da atividade das hidrólases (lactose), défices enzimáticos (frutanos, galactanos) ou a moléculas de elevadas dimensões incapazes de ser absorvidas por difusão simples (polióis) $(3,5,10,16)$.

Este grupo de hidratos de carbono, quando não absorvidos, mobilizam a água para o intestino delgado, por efeito osmótico $(7,14,16,17)$. Quando atingem o cólon, são fermentados por bactérias levando à produção de ácidos orgânicos e gases como o hidrogénio $\left(\mathrm{H}_{2}\right)$, dióxido de carbono $\left(\mathrm{CO}_{2}\right)$ e metano $\left(\mathrm{CH}_{4}\right)$ induzindo distensão abdominal $(4,10$, 17). Para além disso, os ácidos orgânicos resultantes da fermentação bacteriana, podem alterar a sensibilidade visceral e atuar ao nível do estado inflamatório da parede intestinal (5). Normalmente, estes processos ocorrem de forma despercebida ou causam sensações abdominais leves. Contudo, em indivíduos com SII que apresentam hipersensibilidade visceral, pode levar a sintomas que apresentam um maior desconforto ao indivíduo nomeadamente dor abdominal $(4,17)$.

\section{Dieta Baixa em FODMAPs na SII}

Segundo alguns investigadores, uma dieta baixa em FODMAPs pode ser recomendada como terapia de primeira linha para indivíduos com SII (18). Estudos comprovam que uma ingestão de até 0,5 g de FODMAPs (com exceção da lactose) por refeição se mostra eficaz no alívio de sintomas como distensão, dor abdominal, flatulência, fadiga, diarreia, em $70 \%$ dos doentes $(3,19,20)$. Tendo em conta que esta dieta é restrita em fibra, pode-se verificar um agravamento do quadro de obstipação em alguns dos doentes com SII (1, 3, 4, 10).

A dieta baixa em FODMAPs é constituída por três fases: restrição, reintrodução e manutenção. Em baixo, encontram-se descritas as diretrizes gerais para a implementação das três fases.

Fase 1: restrição, num período de quatro a seis semanas, de alimentos com elevado teor de FODMAPs e substituição destes por alimentos com baixo teor (Tabela 1) $(4,14,21)$. Desta forma, a dieta torna-se mais variada diminuindo o risco de deficiências nutricionais $(3,10)$.

Fase 2: reintrodução gradual e isolada de grupo de alimentos anteriormente restritos, por um período de seis a oito semanas, de modo a identificar a tolerância individual para cada tipo de hidratos de carbono com o objetivo de controlar os sintomas a longo prazo $(3,7,10,14)$.

Fase 3: manutenção da dieta com um consumo dos subgrupos de FODMAPs tolerados pelo indivíduo, a longo prazo, de forma a controlar os sintomas $(10,14)$.

Após a introdução desta dieta espera-se uma melhora sintomática entre uma a oito semanas e caso não se verifique nenhuma resposta positiva, a dieta deverá ser interrompida. É importante referir que a não adesão parcial ou total à dieta constitui a causa mais comum da não resposta $(10,21)$.

\section{Dieta Baixa em FODMAPs vs. Microbiota Intestinal}

A adoção de uma dieta baixa em FODMAPs reduz os prebióticos e os substratos fermentáveis disponíveis podendo alterar negativamente a estrutura e função da microbiota intestinal (22-24).

Estudos relatam modificações ao nível da microbiota intestinal após o cumprimento de uma dieta baixa em FODMAPs da qual ocorreu uma diminuição na abundância total das bactérias, sem se registar variações ao nível da diversidade bacteriana $(22,23,25)$.

Esta diminuição verificou-se principalmente ao nível das bactérias benéficas (funções anti-inflamatórias e imunológicas) para o organismo, nomeadamente Bifidobacterium, Akkermansia muciniphila, Faecalibacterium prausnitzii e Lactobacillus responsáveis não só pela manutenção da mucosa como na produção de butirato (20, 23-25). Quantidades inferiores de Bifidobacterium na SII estão associadas inversamente a limiares de dor, indicando que a composição da microbiota intestinal pode estar relacionada com a resposta dos sintomas $(12,26)$.

\section{ANÁLISE CRÍTICA E CONCLUSÕES}

Estudos revelam um benefício de uma dieta baixa em FODMAPs no tratamento dos sintomas da SII, contudo ainda não são claros os efeitos da sua aplicação na saúde gastrointestinal. Ainda assim sabe-se que este padrão alimentar altera a composição da microbiota intestinal sendo as suas implicações (positivas ou negativas) desconhecidas (3). Relativamente aos ensaios clínicos que comprovam a eficácia dos resultados do baixo consumo de FODMAPs existem algumas limitações, tais como a escolha do placebo, avaliação a curto prazo e se o ensaio clínico não for totalmente cego pondo em causa a observação e a conduta do mesmo. Para além disso, não é possível afirmar que a adoção desta dieta a longo prazo trará os benefícios descritos nos ensaios clínicos uma vez que, sendo uma dieta tão restritiva, não é fácil de manter tanto a nível social como de trabalho, apresentando também a desvantagem de ser dispendiosa (4).

A prescrição deste regime alimentar não é fácil, sendo necessária a intervenção de um nutricionista com conhecimento nesta área já que, a incorreta instrução assim como o não cumprimento por parte do doente, a longo prazo, podem levar a um desequilíbrio nutricional agravando o estado de saúde do indivíduo. Contudo, a sua correta realização traduz-se num baixo risco de inadequação nutricional uma vez que nenhum grupo de alimentos é excluído, existindo várias

Tabela 1

Alimentos com elevado teor em FODMAPs e fontes alternativas de alimentos com baixo teor $(4,14,21)$

\begin{tabular}{lll}
\multicolumn{1}{c}{ GRUPO } & \multicolumn{1}{c}{ ALIMENTOS cOM ELEVADO TEOR EM FODMAPS } & \multicolumn{1}{c}{ ALIMENTOS COM BAIXO TEOR EM FODMAPS } \\
$\begin{array}{l}\text { Produtos Hortícolas/Leguminosas/Cogumelos } \\
\text { (Frutose, Oligossacarídeos e Polióis) }\end{array}$ & $\begin{array}{l}\text { Espargos, alcachofras, alho, cebola, beterraba, couve-de- } \\
\text { bruxelas, brócolos, couve-flor, cogumelos, ervilhas, funcho, } \\
\text { lentilhas, repolho, grão-de-bico }\end{array}$ & $\begin{array}{l}\text { Cenoura, espinafres, batata, pepino, beringela, courgette, } \\
\text { abóbora, alface, nabo, milho, pimento, acelga, salsa, feijão- } \\
\text { verde }\end{array}$ \\
\hline $\begin{array}{l}\text { Laticínios } \\
\text { (Lactose) }\end{array}$ & $\begin{array}{l}\text { Leite, iogurte, queijo macio e fresco (ricota, creme de queijo, } \\
\text { cottage) }\end{array}$ & $\begin{array}{l}\text { Leite sem lactose, iogurte sem lactose, queijo duro (brie, } \\
\text { camembert, feta), proteína de soja, bebida de arroz }\end{array}$ \\
\hline $\begin{array}{l}\text { Frutas } \\
\text { (Frutose, Oligossacarídeos, Polióis) }\end{array}$ & $\begin{array}{l}\text { Maçã, pera, melancia, figo, manga, fruta de lata, nectarinas, } \\
\text { pêssego, damascos, ameixas, abacate, cereja, ameixas } \\
\text { secas, lichia) }\end{array}$ & $\begin{array}{l}\text { Laranja, tangerina, kiwi, ananás, morango, mirtilo, } \\
\text { framboesa, limão, lima, uvas, banana, melão, maracujá, } \\
\text { mamão, tomate }\end{array}$ \\
\hline $\begin{array}{l}\text { Pães e cereais } \\
\text { (Oligossacarídeos) }\end{array}$ & $\begin{array}{l}\text { Cevada, centeio, trigo quando consumido em elevadas } \\
\text { quantidades (pão, massa, cuscuz, bolachas) }\end{array}$ & $\begin{array}{l}\text { Pão sem glúten, aveia, massa sem glúten, arroz, quinoa, } \\
\text { bolachas sem glúten }\end{array}$ \\
\hline $\begin{array}{l}\text { Sementes e nozes } \\
\text { (Oligossacarídeos) }\end{array}$ & Pistache, castanha-de-caju, avelã & $\begin{array}{l}\text { Amêndoas }(<10), \text { sementes de girassol, sementes de } \\
\text { sésamo, sementes de abóbora }\end{array}$ \\
\hline
\end{tabular}


alternativas de alimentos com baixo teor de FODMAPs para todos os alimentos com um alto teor (25).

A personalização desta dieta possibilita o empowerment do indivíduo, o controlo a longo prazo dos sintomas e consequente melhoria da qualidade de vida. Alguns investigadores recomendam a dieta baixa em FODMAPs como terapia de primeira linha para doentes com SII (18).

\section{REFERÊNCIAS BIBLIOGRÁFICAS}

1. Passos M do CF, Moraes-Filho JP. Microbiota intestinal nas doenças digestivas. Arq Gastroenterol. 2017;54(3):255-62.

2. Choung RS, Saito YA. Epidemiology of Irritable Bowel Syndrome. GI Epidemiol Dis Clin Methodol Second Ed. 2014;222-34.

3. Ireton-Jones C. The low FODMAP diet: Fundamental therapy in the management of irritable bowel syndrome. Curr Opin Clin Nutr Metab Care. 2017;20(5):414-9.

4. Molina-Infante J, Serra J, Fernandez-Bañares F, Mearin F. The low-FODMAP diet for irritable bowel syndrome: Lights and shadows. Gastroenterol y Hepatol (English Ed. 2016;39(2):55-65.

5. Jouët $P$, Morel N. Low FODMAPs diet, what nutritionist should know about it. Nutr Clin Metab [Internet]. 2019;33(3):199-204. Available from: https://doi.org/10.1016/j. nupar.2019.05.005.

6. Hellström PM. Pathophysiology of the irritable bowel syndrome - Reflections of today. Best Pract Res Clin Gastroenterol. 2019;40-41.

7. Ooi SL, Correa D, Pak SC. Probiotics, prebiotics, and low FODMAP diet for irritable bowel syndrome - What is the current evidence? Complement Ther Med [Internet]. 2019;43(January):73-80. Available from: https://doi.org/10.1016/j.ctim.2019.01.010 8. Soares RLS. Irritable bowel syndrome: A clinical review. World J Gastroenterol. 2014;20(34):12144-60.

9. Principi AN, Cozzali R, Farinelli E, Brusaferro A, Esposito S, Esposito S. Emeritus Professor of Pediatrics, Università degli Studi di Milano, Milan, Italy; 2 Pediatric Correspondence and requests for reprints should be addressed to: J Infect [Internet]. 2017; Available from: https://doi.org/10.1016/j.jinf.2017.12.013.

10. Mehtab W, Agarwal A, Singh N, Malhotra A, Makharia GK. All that a physician should know about FODMAPs. Indian J Gastroenterol. 2019;38(5):378-90.

11. Almeida, Luciana Barros; Marinho, Célia Bastos; Souza, Cristiane da Silva; Cheib VBP. Disbiose Intestinal. Rev bras nutr clín. 2009;24(1):58-65.

12. Wang L, Alammar N, Singh R, Nanavati J, Song Y, Chaudhary R, et al. Gut Microbial Dysbiosis in the Irritable Bowel Syndrome: A Systematic Review and Meta-Analysis of Case-Control Studies. J Acad Nutr Diet [Internet]. 2020;120(4):565-86. Available from: https://doi.org/10.1016/j.jand.2019.05.015.

13. Ispiryan L, Zannini E, Arendt EK. Characterization of the FODMAP-profile in cerealproduct ingredients. J Cereal Sci [Internet]. 2020;92:102916. Available from: https:// doi.org/10.1016/j.jcs.2020.102916.

14. Zugasti Murillo A, Estremera Arévalo F, Petrina Jáuregui E. Diet low in fermentable oligosaccharides, disaccharides, monosaccharides and polyols (FODMAPs) in the treatment of irritable bowel syndrome: Indications and design. Endocrinol y Nutr (English Ed [Internet]. 2016;63(3):132-8. Available from: http://dx.doi.org/10.1016/j. endoen.2015.10.014.

15. Eswaran S, Farida JP, Green J, Miller JD, Chey WD. Nutrition in the management of gastrointestinal diseases and disorders: the evidence for the low FODMAP diet. Curr Opin Pharmacol [Internet]. 2017;37:151-7. Available from: http://dx.doi.org/10.1016/j. coph.2017.10.008.

16. Baranguán Castro ML, Ros Arnal I, García Romero R, Rodríguez Martínez G, Ubalde Sainz E. Implementation of a low FODMAP diet for functional abdominal pain. An Pediatría (English Ed [Internet]. 2019;90(3):180-6. Available from: https://doi. org/10.1016/j.anpede.2018.02.014.

17. Staudacher HM, Whelan K. The low FODMAP diet: Recent advances in understanding its mechanisms and efficacy in IBS. Vol. 66, Gut. 2017. 1517-1527 p. 18. Zhou SY, Gillilland M, Wu X, Leelasinjaroen P, Zhang G, Zhou H, et al. FODMAP diet modulates visceral nociception by lipopolysaccharide-mediated intestinal inflammation and barrier dysfunction. J Clin Invest. 2018;128(1):267-80.
19. Staudacher HM, Ralph FSE, Irving PM, Whelan K, Lomer MCE. Nutrient Intake, Diet Quality, and Diet Diversity in Irritable Bowel Syndrome and the Impact of the Low FODMAP Diet. J Acad Nutr Diet [Internet]. 2020;120(4):535-47. Available from: https:// doi.org/10.1016/j.jand.2019.01.017.

20. Zannini E, Arendt EK. Low FODMAPs and gluten-free foods for irritable bowel syndrome treatment: Lights and shadows. Food Res Int [Internet]. 2018;110:33-41. Available from: http://dx.doi.org/10.1016/j.foodres.2017.04.001.

21. Andrade VLA, Fonseca TN, Gouveia CA, Kobayashi TG, Leite RGS, Mattar RA, et al. Dieta restrita de FODMEPs como opção terapêutica na síndrome do intestino irritável: Revisão sistemática. GED - Gastrenterologia Endosc Dig. 2015;34(1):34-41. 22. Zhan $Y$ le, Zhan $Y$ an, Dai S xue. Is a low FODMAP diet beneficial for patients with inflammatory bowel disease? A meta-analysis and systematic review. Clin Nutr [Internet]. 2018;37(1):123-9. Available from: http://dx.doi.org/10.1016/j.clnu.2017.05.019.

23. Cox SR, Lindsay JO, Fromentin S, Stagg AJ, McCarthy NE, Galleron N, et al. Effects of Low FODMAP Diet on Symptoms, Fecal Microbiome, and Markers of Inflammation in Patients With Quiescent Inflammatory Bowel Disease in a Randomized Trial. Gastroenterology [Internet]. 2020;158(1):176-188.e7. Available from: https://doi. org/10.1053/j.gastro.2019.09.024.

24. Laatikainen R, Jalanka J, Loponen J, Hongisto S-M, Hilliä M, Koskenpato J, et al. Randomised clinical trial: effect of low-FODMAP rye bread versus regular rye bread on the intestinal microbiota of irritable bowel syndrome patients: association with individual symptom variation. BMC Nutr. 2019;5(1):1-11.

25. Gibson PR, Halmos EP. FODMAPs and carbohydrate intolerance [Internet]. Clinical and Basic Neurogastroenterology and Motility. Elsevier Inc.; 2020. 371-386 p. Available from: https://doi.org/10.1016/B978-0-12-813037-7.00026-1.

26. Valeur J, Småstuen MC, Knudsen T, Lied GA, Røseth AG. Exploring Gut Microbiota Composition as an Indicator of Clinical Response to Dietary FODMAP Restriction in Patients with Irritable Bowel Syndrome. Dig Dis Sci [Internet]. 2018;63(2):429-36. Available from: https://doi.org/10.1007/s10620-017-4893-3. 